\title{
VARIABILIDADE GENÉTICA DE PROCEDÊNCIAS E PROGÊNIES DE UMBUZEIRO VIA METODOLOGIA DE MODELOS LINEARES MISTOS (REML/BLUP) ${ }^{1}$
}

\author{
VISÊLDO RIBEIRO DE OLIVEIRA² ${ }^{2}$, MARCOS DEON VILELA DE RESENDE ${ }^{3}$, CLÓVIS EDUARDO DE SOUZA \\ NASCIMENTO ${ }^{4}$, MARCOS ANTÔNIO DRUMOND ${ }^{2}$, CARLOS ANTÔNIO FERNANDES SANTOS ${ }^{5}$
}

\begin{abstract}
RESUMO - O umbuzeiro (Spondias tuberosa Arruda), espécie nativa do semi-árido brasileiro, é um dos recursos genéticos mais importantes para a produção de frutos destinados ao consumo in natura e à industrialização. No melhoramento de espécies perenes, o uso de técnicas de avaliação genética com base em modelos mistos do tipo REML/BLUP (máxima verossimilhança restrita/melhor predição linear não viciada) é fundamental para a predição de valores genéticos aditivos e genotípicos de indivíduos com potencial para seleção, tanto em nível intrapopulacional como interpopulacional. Este procedimento vem sendo aplicado com sucesso no Brasil, no melhoramento de várias espécies frutíferas e florestais. O presente trabalho teve como objetivos estudar a variabilidade genética, estimar parâmetros genéticos e realizar a predição de valores genéticos dos indivíduos, utilizando a metodologia REML/BLUP a partir da avaliação de procedências e progênies de umbuzeiro. O ensaio foi instalado em delineamento de blocos ao acaso, com arranjo hierárquico desbalanceado, constituído de três procedências e 42 progênies. Foram avaliados os caracteres altura de plantas (ALP), maior diâmetro de copa (MAC), menor diâmetro de copa (MEC), diâmetro do colo (DIC) e número de ramos primários (NRP), os quais são correlacionados à produção de frutos. Os resultados obtidos permitem afirmar que, no umbuzeiro, aos nove anos de idade, a maior parte da variabilidade genética encontrada concentra-se dentro de populações. Os caracteres MEC e MAC apresentaram, respectivamente, valores de herdabilidade individual no sentido restrito de 0,08 e 0,14 e ganhos de $6 \%$ e $9 \%$, respectivamente, com a seleção dos dez melhores genitores.
\end{abstract}

Termos para indexação: Spondias tuberosa Arruda, análise genética, predição de ganhos, Anacardiaceae.

\section{GENETIC VARIABILITY OF PROVENANCES AND PROGENIES OF UMBU TREE BY MIXED LINEAR MODEL METHODOLOGY (REML/BLUP)}

\begin{abstract}
The umbu tree, a native species from the Brazilian semi-arid, is one of the most important genetic resources for fruit production in the region. In the improvement of perennial plant species, the use of the mixed model methodology trough the REML/BLUP (restricted maximum likelihood/best linear unbiased prediction) procedure is an essential feature for selecting individuals based on their predicted genetic additive and genotypic values, at the intra and inter population levels. This procedure is being successfully applied to breeding of several fruit and forest tree species in Brazil. This paper aimed to study the genetic variability, estimating genetic parameters and predicting individual breeding values in the umbu tree, through the REML/BLUP procedure applied in the evaluation of populations and progenies. The trial was set out in a randomized complete block design with 42 progenies arranged as a unbalanced hierarchy within 3 populations. The traits plant height (ALP), biggest canopy diameter (MAC), smallest canopy diameter (MEC), base diameter (DIC) and number of primary branches (NRP) were evaluated. These traits are related to fruit production. The results showed that the greatest part of the genetic variability is concentrated within populations. The heritability coefficients were of the order of 0.08 and 0.14 for the traits MEC and MAC, respectively. Genetic gain estimates with selection of the best ten parents were in the order of $6 \%$ and $9 \%$ for MEC and MAC, respectively.
\end{abstract}

Index terms: Spondias tuberosa Arruda, genetic analysis, predicting gain, Anacardeaceae.

\section{INTRODUÇÃO}

O umbuzeiro (Spondias tuberosa Arruda), da família Anacardiaceae, é uma árvore que atinge cerca de $5 \mathrm{~m}$ de altura, exibindo uma copa de até $15 \mathrm{~m}$ de diâmetro (Silva et al., 1979). Segundo Lorenzi (1992), a espécie distribui-se naturalmente nas regiões secas do Nordeste brasileiro, desde o Estado do Ceará até o norte de Minas Gerais.

Estudos relacionados com a conservação ex situ, juntamente com a adoção de práticas de manejo e melhoramento, são alternativas importantes para a sobrevivência do umbuzeiro no semi-árido. Dentro desse contexto, e visando à conservação da variabilidade genética da espécie, Nascimento et al.(2002) implantaram, em 1994, o Banco Ativo de Germoplasma de Umbuzeiro (BGU), na Embrapa Semi-Árido, constituído atualmente de 78 acessos, de genótipos clonados, através de enxertia, de várias regiões agroecológicas do Nordeste. A utilização adequada desses materiais genéticos e futuros estudos de hibridação de genótipos divergentes, no banco de germoplasma, poderão fornecer informações importantes para o melhoramento da espécie no semi-árido brasileiro.

Relatos de Duque (1980) mostram a possibilidade de melhoramento no tamanho do fruto, quantidade de polpa, redução no tamanho do caroço e espessura da casca, através da aplicação de seleção e multiplicação do material pela utilização de enxertia. Porém, faltam informações sobre a estrutura e o padrão de variação genética da espécie, sem as quais, pode-se colocar em risco um programa de melhoramento genético a longo prazo (Guries \& Ledig, 1982; Silva et al., 1987).

Estudos realizados por Silva et al. (1987), sobre caracteres qualitativos e quantitativos dos frutos do umbuzeiro, sugerem a existência de variabilidade entre indivíduos. Santos (1997), realizando estudos sobre a dispersão da variabilidade do umbuzeiro, caracterizou 340 árvores e concluiu que os caracteres peso de fruto, diâmetro de fruto, peso da casca, maior diâmetro de copa e altura de plantas foram os de menor importância para a discriminação fenotípica das árvores avaliadas.

Segundo Kageyama \& Dias (1982), as variações fenotípicas e genéticas dentro e entre populações, para diferentes caracteres, são as formas mais apropriadas para determinar-se a estrutura genética de uma população, desde que as sementes coletadas de indivíduos e/ou populações representativas sejam testadas em condições de laboratório, viveiro ou campo, com o controle de efeitos ambientais adequados.

Segundo Resende (2000), no melhoramento de espécies perenes, o uso de técnicas de avaliação genética, com base em modelos mistos do tipo REML/BLUP (máxima verossimilhança restrita/melhor predição linear não viciada), são fundamentais para a predição de valores genéticos aditivos e genotípicos de indivíduos com potencial para

\footnotetext{
${ }^{1}$ (Trabalho 096/2003). Recebido: 05/08/2003. Aceito para publicação: 06/02/2004.

${ }^{2}$ Eng. Ftal.; PhD. Embrapa Semi-Árido C. P. 23, CEP 56302-970 - Petrolina-PE - Fone: 0xx8738621711- e-mail: v iseldo@cpatsa.embrapa.br.

${ }^{3}$ Eng. Agr.; PhD. Embrapa Florestas C. P. 319, CEP 83411-000 - Colombo-PR - Fone: 0xx416661313 - e-mail: deon@cnpf.embrapa.br.

${ }^{4}$ Eng. Ftal.; MSc. Embrapa Semi-Árido C. P. 23, CEP 56302-970 - Petrolina-PE - Fone: 0xx8738621711- e-mail: clovisen@cpatsa.embrapa.br.

${ }^{2}$ Eng. Ftal.; PhD. Embrapa Semi-Árido C. P. 23, CEP 56302-970 - Petrolina-PE - Fone: 0xx8738621711- e-mail: drumond@cpatsa.embrapa.br.

${ }^{5}$ Eng.Agr.; PhD. Embrapa Semi-Árido C. P. 23, CEP 56302-970 - Petrolina-PE - Fone: 0xx8738621711- e-mail: casantos@cpatsa.embrapa.br.
} 
seleção, tanto em nível intrapopulacional como interpopulacional. O procedimento REML/BLUP vem sendo aplicado com sucesso no melhoramento de fruteiras no Brasil, em espécies como a pupunheira (Farias Neto \& Resende, 2001), a aceroleira (Paiva et al., 2002), o cacaueiro (Resende \& Dias, 2000), a seringueira (Costa et al., 2000) e espécies florestais (Resende et al., 1996).

O presente trabalho teve como objetivos estudar a variabilidade genética, estimar parâmetros genéticos e realizar a predição de valores genéticos dos indivíduos para fins de seleção, utilizando a metodologia REML/BLUP a partir da avaliação de procedências e progênies de umbuzeiro.

\section{MATERIAL E MÉTODOS}

$\mathrm{O}$ experimento foi instalado em área pertencente à Embrapa Semi-Árido, localizada no campo experimental da caatinga, no município Petrolina-PE, a $09^{\circ} 09^{\prime}$ de latitude Sul e $40^{\circ} 22^{\prime}$ de longitude Oeste, com altitude de 365,5m (Embrapa, 1979).

O clima da região é do tipo Bsh, com precipitação média anual de $400 \mathrm{~mm}$, temperatura média anual de 24 a $27^{\circ} \mathrm{C}$ e a umidade relativa de 60 a $70 \%$ (Embrapa, 1979).

O delineamento experimental adotado foi o de blocos ao acaso, com arranjo hierárquico de subparcelas dentro de parcelas, similar a parcelas subdivididas, denominado "compact family block", conforme Wright (1978), constituído de três procedências, representadas pelos municípios de Petrolina-PE, com 14 progênies, Ouricuri-PE com 11 progênies e Massaroca-BA com 17 progênies, totalizando 42 progênies amostradas ao acaso. As parcelas foram representadas pelas procedências e as subparcelas pelas progênies, com arranjo linear, constituídas de 5 plantas, em espaçamento de $8 \mathrm{~m} \times 3 \mathrm{~m}$, com cinco repetições. Os blocos ou repetições representam estratos homogêneos na área experimental e contemplam todas as procedências nas parcelas e todas as progênies nas subparcelas.

Os caracteres analisados aos nove anos de idade foram: altura de plantas (ALP), maior diâmetro de copa (MAC), menor diâmetro de copa (MEC), diâmetro do colo (DIC) e número de ramos primários (NRP). A análise estatística foi baseada em modelos mistos do tipo REML/ BLUP, conforme Resende (2000; 2002). O modelo empregado foi:

\section{Modelo linear misto:}

$\mathrm{y}=\mathrm{Xb}+\mathrm{Za}+\mathrm{Wc}+\mathrm{Tp}+\mathrm{e}$

em que:

$\mathrm{y}=$ vetores de dados;

$\mathrm{b}=$ vetores dos efeitos de blocos (fixos);

$\mathrm{a}=$ vetores dos efeitos genéticos aditivos (aleatórios);

$\mathrm{c}=$ vetores de efeitos de parcelas (aleatórios);

$\mathrm{p}=$ vetores dos efeitos de procedências (aleatórios);

$\mathrm{e}=$ vetores dos erros aleatórios.

$\mathrm{X}, \mathrm{Z}, \mathrm{W}$ e T: matrizes de incidência para b, a, c e p, respectivamente.

Distribuições e estruturas de médias e variâncias:

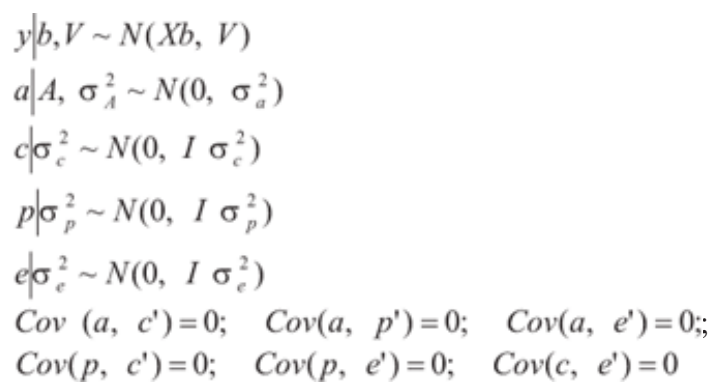

ou seja:

$$
\mathrm{E}\left[\begin{array}{c}
\mathrm{y} \\
\mathrm{a} \\
\mathrm{c} \\
\mathrm{p} \\
\mathrm{e}
\end{array}\right]=\left[\begin{array}{c}
\mathrm{Xb} \\
0 \\
0 \\
0 \\
0
\end{array}\right] ; \quad \mathrm{Var}\left[\begin{array}{c}
\mathrm{y} \\
\mathrm{a} \\
\mathrm{c} \\
\mathrm{p} \\
\mathrm{e}
\end{array}\right]=\left[\begin{array}{ccccc}
\mathrm{V} & \mathrm{ZG} & \mathrm{WC} & \mathrm{TP} & \mathrm{R} \\
\mathrm{GZ} & \mathrm{G} & 0 & 0 & 0 \\
\mathrm{CW}^{\prime} & 0 & \mathrm{C} & 0 & 0 \\
\mathrm{PT}^{\prime} & 0 & 0 & \mathrm{P} & 0 \\
\mathrm{R} & 0 & 0 & 0 & \mathrm{R}
\end{array}\right] \text {, em que: }
$$

$$
\begin{aligned}
& G=A \sigma_{a}^{2} \\
& R=I \quad \sigma_{c}^{2} \\
& C=I \quad \sigma_{c}^{2} \\
& \mathrm{P}=\mathrm{I} \sigma_{\mathrm{p}}^{2} \\
& \mathrm{~V}=\mathrm{ZA} \quad \sigma_{\mathrm{a}}^{2} \mathrm{Z}^{\prime}+\mathrm{WI} \sigma_{\mathrm{c}}^{2} \mathrm{~W}^{\prime}+\mathrm{TI} \quad \sigma_{\mathrm{p}}^{2} \mathrm{~T}^{\prime}+\mathrm{I} \sigma_{\mathrm{e}}^{2} .
\end{aligned}
$$

\section{Equações de modelo misto:}

$$
\begin{gathered}
{\left[\begin{array}{cccc}
X^{\prime} X & X^{\prime} Z & X^{\prime} W & X^{\prime} T \\
Z^{\prime} X & Z^{\prime} Z+A^{-1} \lambda_{1} & Z^{\prime} W & Z^{\prime} T \\
W^{\prime} X & W^{\prime} Z & W^{\prime} W+I \lambda_{2} & W^{\prime} T \\
T^{\prime} X & T^{\prime} Z & T^{\prime} W & T^{\prime} T+I \lambda_{3}
\end{array}\right]\left[\begin{array}{c}
\hat{b} \\
\hat{a} \\
\hat{c} \\
\hat{p}
\end{array}\right]=\left[\begin{array}{c}
X^{\prime} y \\
Z^{\prime} y \\
W^{\prime} y \\
T^{\prime} y
\end{array}\right], \text { em que: }} \\
\lambda_{1}=\frac{1-\rho}{\mathrm{h}^{2}}=\frac{\sigma_{e}^{2}}{\sigma_{\mathrm{a}}^{2}} \quad \lambda_{2}=\frac{1-\rho}{\mathrm{c}^{2}}=\frac{\sigma_{c}^{2}}{\sigma_{\mathrm{c}}^{2}} ; \quad \lambda_{3}=\frac{1-\rho}{\mathrm{p}^{2}}=\frac{\sigma_{c}^{2}}{\sigma_{\mathrm{p}}^{2}} . \\
h^{2}=\frac{\sigma_{a}^{2}}{\sigma_{a}^{2}+\sigma_{c}^{2}+\sigma_{p}^{2}+\sigma_{e}^{2}}: \text { herdabilidade individual no sentido restrito, } \\
\text { no bloco, dentro de procedência; }
\end{gathered}
$$

Estimadores iterativos dos componentes de variância por REML via algoritmo EM:

$$
\begin{aligned}
& \hat{\sigma}_{e}^{2}=\left[\begin{array}{lll}
y^{\prime} y-\hat{b}^{\prime} & X^{\prime} y-\hat{a}^{\prime} \quad Z^{\prime} y-\hat{c}^{\prime} \quad W^{\prime} y-\hat{p}^{\prime} T^{\prime} y
\end{array}\right] /[N-r(x)] \\
& \hat{\sigma}_{a}^{2}=\left[\hat{a}^{\prime} A^{-1} \hat{a}+\hat{\sigma}_{e}^{2} \operatorname{tr}\left(A^{-1} C^{22}\right)\right] / q \\
& \hat{\sigma}_{c}^{2}=\left[\hat{c}^{\prime} c+\hat{\sigma}_{e}^{2} \operatorname{tr} C^{33}\right] / s \\
& \hat{\sigma}_{p}^{2}=\left[\hat{p}^{\prime} \hat{p}+\hat{\sigma}_{e}^{2} \operatorname{tr} C^{44}\right] / q, \text { em que: }
\end{aligned}
$$

$\mathrm{C}^{22}, \mathrm{C}^{33}$ e $\mathrm{C}^{44}$ advém de:

$$
C^{-1}=\left[\begin{array}{llll}
C_{11} & C_{12} & C_{13} & C_{14} \\
C_{21} & C_{22} & C_{23} & C_{24} \\
C_{31} & C_{32} & C_{33} & C_{34} \\
C_{41} & C_{42} & C_{43} & C_{44}
\end{array}\right]^{-1}=\left[\begin{array}{llll}
C^{11} & c^{12} & c^{13} & c^{14} \\
C^{21} & C^{22} & c^{23} & C^{24} \\
C^{31} & C^{32} & C^{33} & C^{34} \\
C^{41} & C^{42} & C^{43} & C^{44}
\end{array}\right]
$$

Os efeitos genéticos aditivos foram obtidos através do uso da matriz de parentesco genético aditivo (A), assumindo o parentesco de meios-irmãos entre os indivíduos de cada progênie de polinização aberta. Isto é adequado, visto que a espécie é alógama. 


\section{RESULTADOS E DISCUSSÃO}

Os valores médios para os cinco caracteres avaliados equivaleram a $1,60 \pm 0,40 ; 3,86 \pm 1,09 ; 3,35 \pm 0,97 ; 6,95 \pm 1,48$ e 2,96 $\pm 1,02$ para os caracteres ALP, MAC, MEC, DIC e NRP, respectivamente. Os três primeiros caracteres foram avaliados em metros, DIC em centímetros e NRP consistiu de simples contagem.

Não foi constatada variabilidade genética para os caracteres altura da planta, diâmetro do colo e número de ramos primários. Santos (1997) também verificou pequena variabilidade para o caráter altura das plantas.

Para os caracteres maior diâmetro da copa (MAC) e menor diâmetro da copa (MEC), os resultados são apresentados na Tabela 1. Constata-se que a variabilidade genética entre procedências foi praticamente nula para todos os caracteres, conduzindo a estimativas nulas de herdabilidade entre procedências. Este fato revela que não é possível a seleção entre procedências para os caracteres na idade avaliada. Por outro lado, existe considerável variabilidade genética dentro de populações, conduzindo a estimativas da herdabilidade individual, no sentido restrito, variando de $8 \%$ a $14 \%$. Apesar de as estimativas de herdabilidade terem sido de baixa magnitude em nível de indivíduo, a seleção individual pelo procedimento BLUP considera também as informações de parentes (informação da família), fato que propicia uma razoável acurácia seletiva. Em geral, herdabilidades individuais de baixa magnitude são comuns para os caracteres quantitativos e, via de regra, conduzem a moderadas magnitudes das herdabilidades em nível de médias de família (Resende, 2002).

TABELA 1 - Estimativas de componentes de variância e parâmetros genéticos para os caracteres maior diâmetro da copa (MAC) e menor diâmetro da copa (MEC), em teste de procedências e progênies de umbuzeiro no semi-árido brasileiro.

\begin{tabular}{lll}
\hline Parâmetro genético & MAC & MEC \\
\hline Herdabilidade individual no sentido restrito $\left(h^{2}\right)$ & 0,1400 & 0,0800 \\
Variância genética aditiva dentro de procedências $\left(\sigma_{a}^{2}\right)$ & 0,1655 & 0,0771 \\
Variância genética entre procedências $\left(\sigma_{p}^{2}\right)$ & 0,0102 & 0,0084 \\
Variância ambiental entre parcelas $\left(\sigma_{c}^{2}\right)$ & 0,2345 & 0,2329 \\
Variância ambiental dentro de parcelas $\left(\sigma_{e}^{2}\right)$ & 0,7486 & 0,6195 \\
Variância fenotípica individual $\left(\sigma_{f}^{2}\right)$ & 1,1597 & 0,9379 \\
Herdabilidade individual entre procedências $\left(p^{2}\right)$ & 0,0000 & 0,0000 \\
\hline
\end{tabular}

A presença de variabilidade genética para MAC e MEC revela também a possibilidade de melhoramento para os caracteres peso total dos frutos por planta e número total de frutos por planta, tendo em vista a correlação positiva envolvendo esses e aqueles caracteres (Santos \& Nascimento, 1998; Santos et al., 1999). Assim, MAC e MEC podem ser usados como uma forma indireta de seleção para a produção de frutos.

As estimativas dos coeficientes de herdabilidade individual, no sentido restrito, embora de baixas magnitudes, revelam excelentes possibilidades de seleção, pois conduziram a estimativas da herdabilidade, no sentido restrito, em nível de médias de família, equivalentes a $38 \%$ e $53 \%$, para os caracteres MEC e MAC, respectivamente. Tais estimativas da herdabilidade em nível de médias de família foram obtidas pela seguinte expressão, conforme Resende (2002): $h_{m}^{2}=\frac{\left[1+(n-1) \rho_{a}\right] h^{2}}{1+(n-1)\left(\rho_{a} h^{2}\right)}$, em que $\rho_{a}$ é a correlação genética entre os indivíduos do tipo de família considerada $\left(\rho_{a}=1 / 4\right.$, para meiosirmãos), $h^{2}$ é a herdabilidade individual no sentido restrito e $n$ é o número total de indivíduos por progênie ( $n$ máximo igual a 25 no presente trabalho). As acurácias seletivas para a seleção de famílias equivaleram a $62 \%$ e $73 \%$ para os caracteres MEC e MAC, respectivamente. Tais acurácias são dadas por: $r_{\text {âam }}=\left[\frac{\left[1+(n-1) \rho_{a}\right] h^{2}}{1+(n-1)\left(\rho_{a} h^{2}\right)}\right]^{1 / 2}$

As herdabilidades para a seleção de genitores, com base no comportamento de suas progênies, equivaleram a $68 \%$ e $95 \%$, para os caracteres MEC e MAC, respectivamente. Tais estimativas da herdabilidade foram obtidas pela seguinte expressão, conforme Resende (2002):

$h_{p}^{2}=\frac{2 n h^{2}}{4+(n-1) h^{2}}$.

Ganhos genéticos da ordem de $6 \%$ e $9 \%$ poderão ser obtidos com a seleção dos 10 melhores genitores para os caracteres MEC e MAC, respectivamente. Os ganhos genéticos foram estimados como a média dos valores genéticos preditos dos genitores selecionados, conforme Resende (2002). As acurácias seletivas para a seleção de genitores equivaleram a $82 \%$ e $97 \%$, para os caracteres MEC e MAC, respectivamente, dadas por:

$r_{\text {alap }}=\left[\frac{2 n h^{2}}{4+(n-1) h^{2}}\right]^{1 / 2}$

Os tamanhos efetivos populacionais associados a cada população equivaleram a: 39 para Ouricuri-PE; 50 para Petrolina-PE; 60 para Massaroca-BA. Estes tamanhos efetivos foram obtidos pela expressão $N_{e}=\frac{4 N_{f} k_{f}}{k_{f}+3}$, conforme Vencovsky (1986), em que $N_{f}$ é o número de matrizes amostradas por população e $k_{f}$ é o número médio de indivíduos amostrados (plantados) por matriz. Uma população composta, obtida pela recombinação de indivíduos selecionados nestas populações, poderá ser adequada para programas de melhoramento a curto e longo prazos, e deverá ser também utilizada como população de conservação ou banco de germoplasma, visando a garantir a utilização futura da espécie, como produtora de frutos.

\section{CONCLUSÕES}

1) A população composta pelas três procedências apresenta bom potencial seletivo e variabilidade genética suficiente para o melhoramento genético a curto e longo prazo.

2) Estimativas iniciais da herdabilidade individual, no sentido restrito, para os caracteres MEC e MAC apresentaram magnitudes baixas.

3) Ganhos genéticos consideráveis podem ser obtidos com a seleção dos 10 melhores genitores para os caracteres MEC e MAC.

4) A acurácia ou precisão na seleção de genitores apresenta magnitudes elevadas.

\section{REFERÊNCIAS BIBLIOGRÁFICAS}

COSTA, R. B.; RESENDE, M. D. V.; ARAÚJO, A. J.; GONÇALVES, P. S.; HIGA, A. R. Selection and genetic gain in rubber tree (Hevea) populations using a mixed mating system. Genetics and Molecular Biology, Ribeirão Preto, v. 23, n. 3, p. 671-679, 2000.

DUQUE, J. G. O Nordeste e as lavouras xerófilas. 3.ed. Mossoró: ESAM, 1980.316p.

EMBRAPA. Centro de Pesquisa Agropecuária do Trópico Semi-Árido. Petrolina-PE. Relatório técnico anual do Centro de Pesquisa Agropecuária do Trópico Semi-Árido, 1977-1978. Brasília: EMBRAPA-DID, 1979. 133p. il.

FARIAS NETO, J. T; RESENDE, M. D. V. de. Aplicação da metodologia de modelos mistos (REML/BLUP) na estimação de componentes de variância e predição de valores genéticos em pupunheira (Bactris gasipaes L.). Revista Brasileira de Fruticultura, Jaboticabal, v. 23, n. 2,p. 320-324, 2001. 
GURIES, R. P. ; LEDIG, F. P. Genetic diversity and population structure in pith pine (Pinus rigida Mill.) Evolution, Lancaster, v.36, n.2, p. 387402, 1982.

KAGEYAMA, P. Y. ; DIAS, I. S. Aplicação da genética em espécies florestais nativas. In: CONGRESSO NACIONAL SOBRE ESSÊNCIAS NATIVAS, 5., 1982, Campos do Jordão, Anais... p. 782-791.

LORENZI, H. Árvores brasileiras: manual de identificação e cultivo de plantas arbóreas nativas do Brasil. Nova Odessa: Ed. Plantarum, 1992.352p.

NASCIMENTO, C. E. S. de; SANTOS, C. A. F.; OLIVEIRA, V. R. de; DRUMOND, M. A. Banco de germoplasma do umbuzeiro: novos acessos e avaliações preliminares aos oito anos de idade. In: CONGRESSOBRASILEIRO DE FRUTICULTURA, 17., 2002, Belém. Anais... Belém: SBF, 2002. CD-ROM.

PAIVA, J. R. de; RESENDE, M. D. V. de; CORDEIRO, E. R. Índice multiefeitos (BLUP) e estimativas de parâmetros genéticos aplicados ao melhoramento da acerola. Pesquisa Agropecuária Brasileira, Brasília, v. 37,n. 6, p. 799-807, 2002.

RESENDE, M. D. V. de. Análise estatística de modelos mistos via reml/ blup na experimentação em melhoramento de plantas perenes. Colombo: Embrapa Florestas, 2000. 101p. (Documentos, 47).

RESENDE, M. D. V. de; DIAS, L. A. S. Aplicação da metodologia de modelos mistos (REML/BLUP) na estimação de parâmetros genéticos e predição de valores genéticos em espécies frutíferas. Revista Brasileira de Fruticultura, Jaboticabal, v. 22, n. 1, p. 44-52, 2000.

RESENDE, M.D.V. de. Genética biométrica e estatística no melhoramento de plantas perenes. Brasília: Embrapa Informação Tecnológica, 2002.975p.
RESENDE, M. D. V. de; PRATES, D. F.; JESUS, A.; YAMADA, C. K. Estimação de componentes de variância e predição de valores genéticos pelo método da máxima verossimilhança restrita (REML) e melhor predição linear não viciada (BLUP) em Pinus. Boletim de Pesquisa Florestal, Colombo, n. 32/33, p. 18-45, jan./dez. 1996.

SANTOS, C. A. F. Dispersão da variabilidade genética do umbuzeiro no semi-árido brasileiro. Pesquisa Agropecuária Brasileira, Brasília, v. 32, n. 6, p. 923-930, 1997.

SANTOS, C. A. F.; NASCIMENTO, C. E. S. Relação entre caracteres quantitativos do umbuzeiro (Spondias tuberosa). Pesquisa Agropecuária Brasileira, Brasília, v. 33, n.4, p. 449-456, 1998.

SANTOS, C.A. F.; NASCIMENTO, C. E. S.; OLIVEIRA, M. C. Recursos genéticos do umbuzeiro: preservação, utilização e abordagem metodológica. In: QUEIRÓZ, M. A; GOEDERT, C. O; RAMOS, S. R. $\mathrm{R}$. (Ed.). Recursos genéticos e melhoramento de plantas para o Nordeste brasileiro. Petrolina: Embrapa Semi-Árido; Brasília: Embrapa Recursos Genéticos e Biotecnologia, 1999. Disponível em: $<$ http://www.cpatsa.embrapa.br.> Acesso em: 30/maio/2003.

SILVA, C. M. M. de S.; PIRES, I. E.; SILVA, H. D. da. Caracterização de frutos do umbuzeiro. Petrolina: EMBRAPA-CPATSA, 1987. 17p. ( Boletim de Pesquisa, 34).

SILVA, C. M. M. de S.; PIRES, I. E.; SILVA, H. D. da. Propagação vegetativa do umbuzeiro. In: CONGRESSO NACIONAL DE BOTÂNICA, 30., 1979, Campo Grande. Anais... Campo Grande: SBB, 1979. p.131-134.

VENCOVSKY, R. Amostragem genética em populações naturais. Silvicultura, São Paulo, v. 41, p. 95-96, 1986.

WRIGHT, J. W. A simplified design for combined provenance and progeny testing. Silvae Genetica, Frankfurt, v. 27, n. 2, p. 68-70, 1978. 